Ewing's sarcoma (ES) and primitive neuroectodermal tumour (PNET) are now considered to be the same tumour and usually occur in long bones. Extraskeletal Ewing's sarcoma is an extremely rare neoplasm, accounting for $1 \%$ of soft tissue sarcomas, with most common location in the thorax. Gallbladder cancer (GBC) represents the most common type among the biliary tract cancers with a poor prognosis even among patients undergoing aggressive therapy.

We present study of extraskeletal ES/ PNET found in the hilus of the liver of an elderly, diagnosed one month prior with GBC woman. The patient underwent two cycles of chemotherapy SAIME/SAVAC for ES and thereafter was operated. During three-year follow-up no recurrence of ES/PNET has been reported. However, two years after chemotherapy the patient suffered a relapse of adenocarcinoma of the gallbladder and thus received palliative chemotherapy of gemcitabine and cisplatin. After 16 months of recurrence she died. To the best of our knowledge, this is the first case of ES/ PNET located in the hilus of the liver and as a synchronous neoplasm.

Key words: extraskeletal Ewing's sarcoma, PNET, hilus of liver, gallbladder carcinoma.

Contemp Oncol (Pozn) 2016; 20 (6): 496-500 DOI: https://doi.org/10.5114/wo.2016.65612

\section{Synchronous extraskeletal Ewing's sarcoma/PNET and gallbladder carcinoma: a case report and literature review}

\author{
Andrzej Plis ${ }^{1 *}$, Aneta Zygulska ${ }^{1}$, Mirosława Püsküllüoğlư ${ }^{1}$, \\ Wojciech Szczepański², Magdalena Białas² ${ }^{2}$, Maciej Krupiński ${ }^{3}$, \\ Krzysztof Krzemieniecki $^{1}$
}

${ }^{1}$ Department of Clinical Oncology, Krakow University Hospital, Krakow, Poland ${ }^{2}$ Department of Clinical and Experimental Pathomorphology, Jagiellonian University Medical College, Krakow, Poland

${ }^{3}$ John Paul II Specialist Hospital, Krakow, Poland

*current employment: Clinic of Hematology and Oncology, Transplant and Palliative Medicine, University Hospital in Greifswald, Greifswald, Germany

\section{Introduction}

The Ewing's sarcoma (ES) family of tumours includes ES and primitive neuroectodermal tumour (PNET), which are primary malignant small round cell tumours of bone and soft tissue. ES and PNET have a similar neuronal phenotype and, because they share an identical chromosome translocation, they should be viewed as two variants of the same tumour that differ only in their degree of neuronal differentiation. Tumours that demonstrate neuronal differentiation are labeled PNETs, and those that are undifferentiated are diagnosed as ES. This distinction has no clinical significance [1]. However, characteristics and outcomes differ among patients with extraskeletal ES/ PNET in comparison with those with skeletal ES/PNET (Table 1) [2-4]. ES/ PNETs represent $1 \%$ of soft tissues sarcomas [2]. This neoplasm has been reported in various locations, but to the best of our knowledge, the case in question is the first case of ES/PNET located in the hilus of liver and as a synchronous neoplasm.

Gallbladder carcinoma represents the most common and aggressive type among the biliary cancers. It occurs two to six times more often in women and its rate increases with age. About $90 \%$ of cases are connected with gallstone disease. The most frequent type of the tumour is adenocarcinoma that accounts for more than $80 \%$ of gallbladder cancers [5]. The simultaneous occurrence of gallbladder adenocarcinoma and other malignancies has been reported only in a few cases involving the coexistence of adenocarcinoma of pancreas, lymphoma of mucosa associated lymphoid tissue of the gallbladder, hepatocellular carcinoma, uterine endometrioid adenocarcinoma and ovarian endometrioid carcinoma [6-9].

\section{Case report}

Laparoscopic simple cholecystectomy was performed in a 70-year old female due to the chronic cholelithiasis. The postoperative abdominal ultrasound was free of any suspicious changes. Pathological examination of the gallbladder specimen revealed the presence of adenocarcinoma. One month later, a hypoechogenic mass of $54 \times 52 \times 43 \mathrm{~mm}$ located in the hilus of the liver was found on control ultrasonography (USG) and computed tomography (CT) of the abdomen (Fig. 1A). Computed tomography of the chest was free of any changes. The patient stayed asymptomatic with performance status (PS) 0 at that time. This lesion was diagnosed as ES/PNET after core biopsy under 
Table 1. Comparison of skeletal and extraskeletal ES clinical features.

\begin{tabular}{|c|c|c|}
\hline & Skeletal ES & Extraskeletal ES \\
\hline Age onset & Most often in $2^{\text {nd }}$ decade & Usually under 35 years, mostly 2 nd and 3rd decade \\
\hline Sex predominance & Male & Male or female \\
\hline Location & Long bones (diaphysis) & $\begin{array}{l}\text { Thorax (32\%), extremities ( } 26 \%) \text {, head and neck (18\%), } \\
\text { retroperitoneum and pelvis (16\%) }\end{array}$ \\
\hline Symptoms & $\begin{array}{l}\text { In } 40 \% \text { patients: pain, enlargement of the extremity, } \\
\text { infection- like with increased body temperature } \\
1 / 4-1 / 3 \text { patients at diagnosis have metastatic disease } \\
\text { (most common in lungs and bones) }\end{array}$ & $\begin{array}{l}\text { In one third of patients pain, polisymptomatic } \\
\text { (depending on the location) }\end{array}$ \\
\hline Treatment & $\begin{array}{l}\text { CTH (chemotherapy) with RTH (radiotherapy) play } \\
\text { main role, surgery as additional treatment }\end{array}$ & $\begin{array}{l}\text { No standards, multimodal considered to be the best } \\
\text { approach - CTH plus local treatment (surgery/RTH) }\end{array}$ \\
\hline 5-year survival rate & $60-70 \%$ when localized, $20-40 \%$ when metastatic & $38-83 \%$ \\
\hline $\begin{array}{l}\text { Negative } \\
\text { prognostic factors }\end{array}$ & $\begin{array}{l}\text { Metastatic disease, size }>5 \mathrm{~cm} \text {, located in pelvis and } \\
\text { chest, infiltrating soft tissues, elevated LDH (lactate } \\
\text { dehydrogenase) }\end{array}$ & $\begin{array}{l}\text { Metastatic disease, elderly, pelvic location, incomplete } \\
\text { resection, tumor size } \geq 8 \mathrm{~cm} \text {, elevated LDH, poor } \\
\text { histological response to CTH, RTH only as local } \\
\text { treatment, positive surgical margins }\end{array}$ \\
\hline $\begin{array}{l}\text { Positive prognostic } \\
\text { factors }\end{array}$ & Female, treatment within 1 month of diagnosis & $\begin{array}{l}\text { Normal level of hemoglobin, LDH, complete response to } \\
\text { CTH, younger age, total resection, type } 1 \text { of the fusion } \\
\text { EWS/FLI 1, located in the extremities, adjuvant RTH }\end{array}$ \\
\hline
\end{tabular}

sonographic control. Skeletal scintigraphy showed no abnormalities. The patient underwent 2 cycles of intravenous chemotherapy according to the protocol of British Columbia Cancer Agency (BCCA) for ES/PNET - SAIME (etoposide $100 \mathrm{mg} / \mathrm{m}^{2}$, mesna $720 \mathrm{mg} / \mathrm{m}^{2}$, ifosfamide $\left.1800 \mathrm{mg} / \mathrm{m}^{2}\right)$ given for 5 consecutive days every 3 weeks alternating with SAVAC (vincristine $1.5 \mathrm{mg} / \mathrm{m}^{2}$, doxorubicin $75 \mathrm{mg} / \mathrm{m}^{2}$, cyclophosphamide $1200 \mathrm{mg} / \mathrm{m}^{2}$ ) given over one day every 3 weeks. Control CT scans after two cycles showed a 57\%

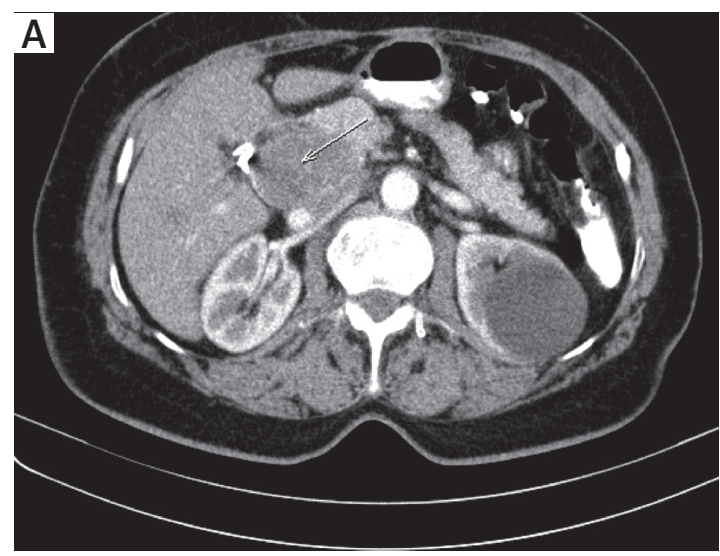

Fig. 1. A) Hepatic tumor infiltrating head of the pancreas, vena cava inferior and portal vein. B) Subsequently performed CT revealed regression of the tumor. C) Tumor infiltrating head of the pancreas, vena cava inferior, portal vein and hepatic artery tumour mass reduction in the biggest dimension (Fig. 1B). Thereafter, the patient was operated and resection of the hilus of the liver, hepato-duodenal ligament and local lymph nodes was performed. The patient stayed asymptomatic under regular control of every 3 months. Two years later, a mass of $46 \times 25 \mathrm{~mm}$ between inferior vena cava, left liver lobe and the head of the pancreas, and a second mass of $17 \times 14 \mathrm{~mm}$ in the common bile duct were revealed on the control CT (Fig. 1C). The patient's PS was 2 and she
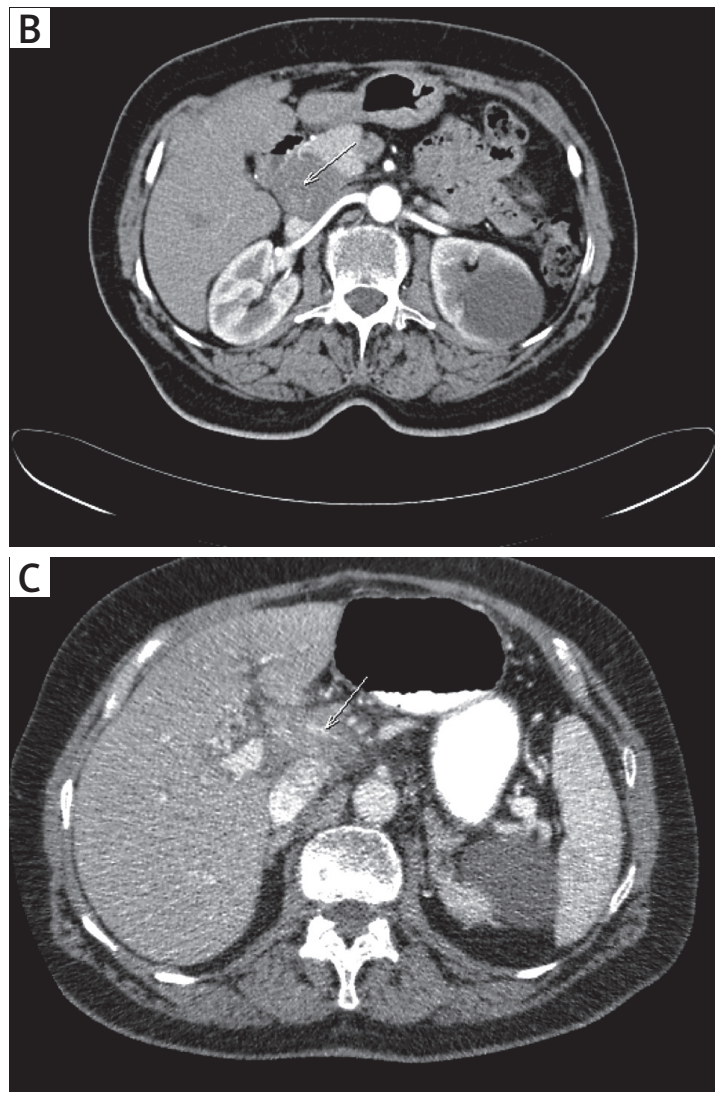
presented with jaundice. She underwent ERCP (endoscopic retrograde cholangio-pancreatography) with insertion of the stent to the common bile duct. Core biopsy revealed the recurrence of gallbladder adenocarcinoma with infiltration of the pancreas. Metastatic lesion was also presented in the liver on subsequently performed control CT. The patient underwent palliative intravenous chemotherapy of cisplatin $\left(25 \mathrm{mg} / \mathrm{m}^{2}\right)$ and gemcitabine $\left(1000 \mathrm{mg} / \mathrm{m}^{2}\right)$ given both on the first and eighth day of the cycle repeated every 3 weeks. Partial regression according to RECIST (Response Evaluation Criteria in Solid Tumours) 1.1 of the lesions located in the hilus of the liver and left liver lobe was observed on control CT scan after 2 cycles of chemotherapy and thus chemotherapy has been continued till 6 cycles. After that time stable disease on control CT of the abdomen has been observed. One month later the patient's PS was 4 and she presented again with jaundice. A flow-restoring procedure was performed on the intra-biliary stent. The patient was admitted to the palliative care department, where she died. No postmortem examination was conducted.

\section{Material and methods}

Biopsy materials were examined with usage of formalin fixed, paraffin wax embedded tissue stained routinely with hematoxilin and eosine (HE). Immunohistochemistry included antibodies to cytokeratine 7 (CK7), cytokeratine 18 (CK18), leucocyte common antigen (LCA), epithelial membrane antigen (EMA), chromogranin, synaptophysin, vimentin, desmin, neurone specific enolase (NSE), thyroid transcription factor (TTF), cluster differentiation 117 (CD117), S-100 protein, Ki67 and MyoD1.

A wide panel of immunohistochemical stainings was performed to exclude the diseases within the scope of the differential diagnosis of Ewing's sarcoma. Briefly, a representative HE section was selected for immunohistochemical investigation and $4 \mu \mathrm{m}$ sections were prepared. The immunohistochemistry was performed by standard method: the slides were de-waxed, rehydrated and incubated in 3\% peroxide solution for 10 minutes to block the endogenous peroxidase activity. Antigen retrieval was carried out by microwaving in citrate buffer $(\mathrm{pH}=6)$ or EDTA $(\mathrm{pH}=8)$ for 5 minutes at $700 \mathrm{~W}$, then for 5 minutes at $600 \mathrm{~W}$. The Lab-Vision detection system was used. The primary antibodies and the respective technical details are summarised in Table 2.

\section{Results}

Removed gallbladder, length $11 \mathrm{~cm}$, with thickened wall, filled with gallstones was sent for the histological examination. This revealed the presence of adenocarcinoma with infiltration of muscularis propria, T1bNxMO (Fig. 2A). An operative margin was free of cancer but a small group of cancer cells was visible in the lumen of the gallbladder at the resection margin. Most probably this finding was irrelevant and the cancer cells were placed there when cutting the material.

Microscopic examination of the lesion located in the hilus of the liver, diagnosed as ES/PNET showed a small cell neoplasm with membranous expression of CD99, NSE, vimentin and synaptophysin. Proliferative activity defined as the percentage of cells positive in Ki67 was higher than 90\%. Staining for CK7, CK18, LCA, EMA, chromogranin, TTF1, CD117 and S-100 protein were negative. Expression of MyoD1 was weak, cytoplasmatic and not characteristic for neoplasms of muscular origin (Fig. 2B-D). After chemotherapy for ES/PNET, examination of the specimens removed within surgery has revealed inflammatory cell infiltration and necrosis with no presence of neoplastic cells. The histological picture together with the results of immunohistochemical reactions were consistent with ES/PNET, which is a highly cellular neoplasm consisting of sheets and nests of small, uniform, round or polygonal cells with scanty cytoplasm and usually high mitotic activity.

\section{Discussion}

Presented case report highlights an extremely rare type of soft tissue sarcoma within atypical location. Unusual is

Table 2. Primary antibodies used for immunohistochemical analysis

\begin{tabular}{|c|c|c|c|c|}
\hline Specificity & Clone & Manufacturer & Dilution & Antigen retrieval \\
\hline CK7 & OV-TL12/30 & DACO & $1: 50$ & citrate buffer \\
\hline CK18 & DC10 & DACO & $1: 50$ & citrate buffer \\
\hline LCA & ZB11+PD7 & DACO & $1: 100$ & citrate buffer \\
\hline EMA & E29 & DACO & $1: 100$ & - \\
\hline chromogranin & DAK-A3 & DACO & $1: 600$ & citrate buffer \\
\hline synaptophysin & SY38 & DACO & $1: 100$ & EDTA \\
\hline vimentin & V9 & DACO & $1: 50$ & citrate buffer \\
\hline desmin & D33 & DACO & $1: 50$ & citrate buffer \\
\hline NSE & BBS/NC/VI/H14 & DACO & $1: 100$ & citrate buffer \\
\hline TTF1 & $8 G 7 G 3 / 1$ & DACO & $1: 500$ & citrate buffer \\
\hline CD117 & policl. & DACO & $1: 100$ & EDTA \\
\hline $\mathrm{S} 100$ & policl. & DACO & $1: 400$ & - \\
\hline Ki-67 & MIB1 & DACO & $1: 200$ & citrate buffer \\
\hline MyoD1 & $5.8 \mathrm{~A}$ & DACO & $1: 50$ & citrate buffer \\
\hline
\end{tabular}



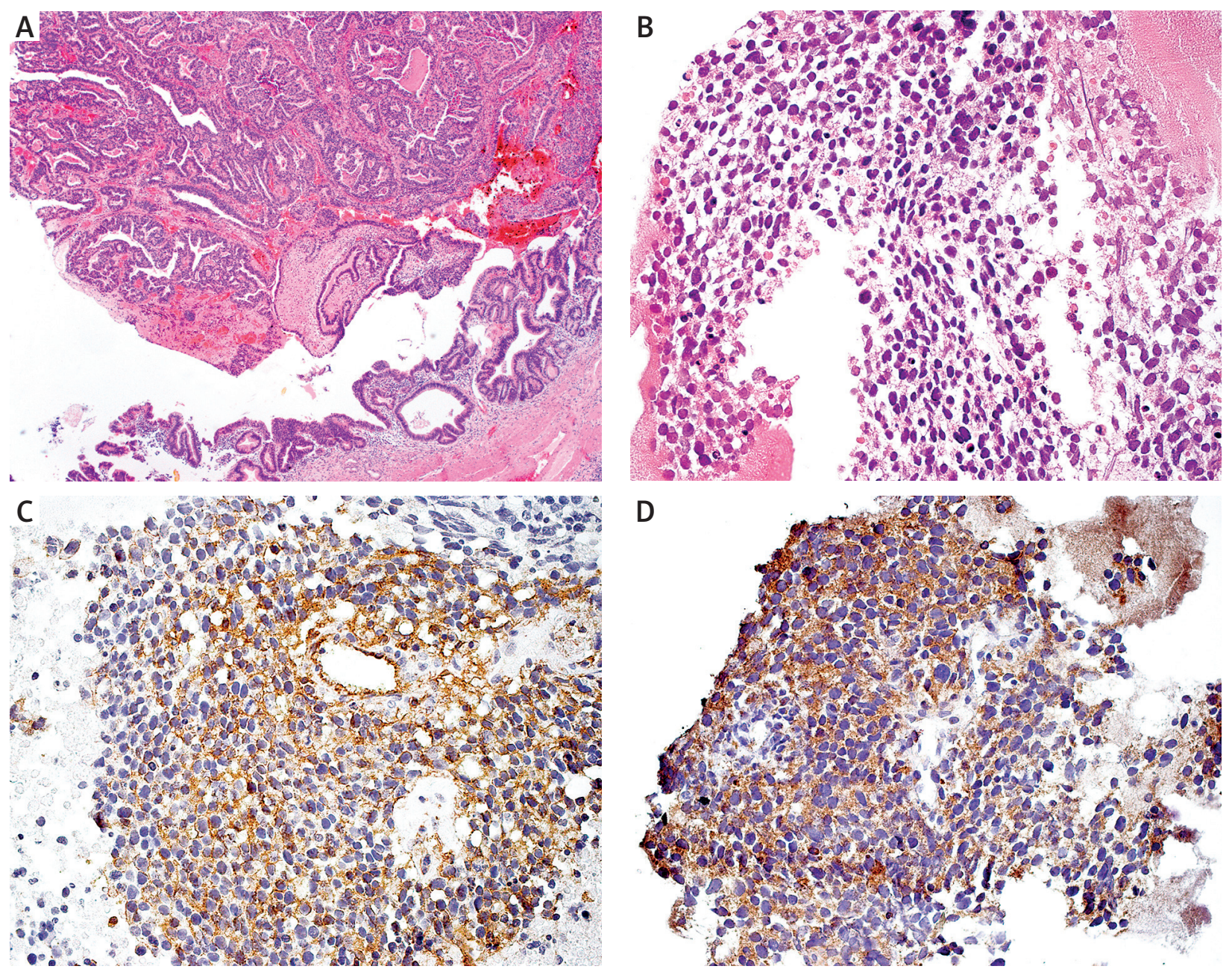

Fig. 2. A) Gallbladder wall with exophytic part of adenocarcinoma; HE, magnification 5x. B) Histological picture of PNET localised within hilus of the liver: sheets of small, uniform, polygonal cells with scanty cytoplasm; HE, magnification 20x; C) Immunohistochemistry for CD99; membranous staining in PNET cells, magnification 20x; D) Immunohistochemistry for synaptophysin: positive, cytoplasmic, granular reaction in PNET cells, magnification 20x

also elderly age of the patient and existence of GBC in the same region as a synchronous neoplasm. Additionally, this case documents pathological diagnosis of ES/PNET, which is based on both histology and immunohistochemistry (the gold standard) and may be problematic due to the rarity of these tumours and no typical location [10]. Core biopsy, as it has been shown, is usually sufficient for making diagnosis. Immunohistochemistry is essential as the family of small round cell tumours is large and includes a lot of different neoplasms, for instance, non-Hodgkin lymphoma, neuroblastoma, alveolar rhabdomyosarcoma, mesenchymal chondrosarcoma, retinoblastoma and desmoplastic small round cell tumour. Membranous expression of CD99 coexisting with positive expression of NSE or synaptophysin are sufficient for the diagnosis of ES/PNET. Other negative immunohistochemical reactions (for instance LCA, MyoD1) are helpful in eliminating neoplasms from the small round cell tumours family $[1,10]$. Genetic studies are useful because translocation $\mathrm{t}(11 ; 22)(\mathrm{q} 24 ; q 12)$ and expression of a fusion gene EWS-FLI1 are specific for ES/PNETs but they are not always available [11]. It should be borne in mind that up to $15 \%$ of ES/PNETs can be negative for this translocation, which is connected with presence of other rare mutations. It is also known that the presence of this translocation is not likely to be considered diagnostic of ES and PNET in the absence of supporting histological evidence[12].

Treatment of EES/PNET usually includes multimodal chemotherapy combined with aggressive surgical treatment or radiotherapy [13]. In contrast to ES/PNET, surgery plays a major role [14]. Multi-agent chemotherapy is superior to single agent one, includes cyclophosphamide, ifosfamide, etoposide, doxorubicin, vincristine, and should be given 12-24 weeks before operation [15]. The presented patient was treated with multimodal chemotherapy of BCCA. Surgery should be performed after neoadjuvant chemotherapy because the probability of achieving complete resection with microscopic free margins is increased. In our case chemotherapy resulted in the regression on CT imaging and finally, necrosis of the neoplasm, which was a sign of good response to the treatment and could be considered as a good prognostic factor. The recommended operation is a wide resection encompassing a rim of $2-3 \mathrm{~cm}$ of normal tissue. When resection is either incomplete or impossible, radiotherapy is an alternative with the same 
dosage as in ES/PNET [14]. The value of adjuvant chemotherapy in clinical improvement remains not clear [15].

About $30-40 \%$ of patients with ES experience recurrence. The rate for patients with EES remains unclear and might be similar [15]. Therapy for refractory or relapsed disease consists of participation in clinical trials, chemotherapy with/without radiotherapy and surgery, if possible. New strategies for the treatment of ES/PNET include stem cell transplantation, antiangiogenic agents (bevacizumab, vinblastine, celecoxib), mTOR pathway inhibition (sirolimus), bisphosphonates, anti-insulin-like growth factor receptor 1 (IGFR1) agents (cixutumumab, R1507) and anti-CD99 agents [16].

At our patient no recurrence of PNET/EES has been reported within 42 months after diagnosis till her death, which was caused by the relapse of the GBC. Complete resection is the only potential curative treatment of GBC, however only $10 \%$ of patients are considered candidates for surgical treatment. Data concerning treatment of T1b stage disease are scant, however National Comprehensive Cancer Network recommends in this case radical cholecystectomy [17]. Even for a patient undergoing aggressive surgery, a 5 -year survival rate is mere $5-10 \%$. Several retrospective reports suggest beneficial effects of both adjuvant and neoadjuvant (chemo)radiotherapy. In relapsed disease, palliative chemotherapy should be considered. Combination of cisplatin and gemcitabine has been used as a standard treatment because it has been shown to increase survival rate (11.7 vs. 8.1 months) comparing with gemcitabine as the only agent [18].

Summing up, this case confirms that EES/PNET is a chemotherapy-sensitive neoplasm. The most beneficial therapy for this tumour remains systemic treatment combined with surgery. New strategies of treatment for EES/PNET are under investigation and thus hopeful. Unfortunately, the overall survival of patients with GBC remains poor and the recurrence rate is still high. That is why this major challenge in oncology requires the initiation of new clinical efforts and trials as well as the integration of the ones conducted previously.

The authors wish to thank Pawet Różanowski and Monika Merdalska, the patient's attending physicians, for access to medical documentation and their valuable remarks regarding the case.

The authors declare no conflict of interest.

\section{References}

1. Rosenberg AE. Bones, Joints and Soft-Tissue Tumors. In: Robbins and Cotran Pathologic Basis of Diseases. Kumar V, Abbas AK, Fausto N, Aster JC. $8^{\text {th }}$ ed. Elsevier Saunders, Philadelphia 2010; 1232-3.

2. Xie CF, Liu MZ, Xi M. Extraskeletal Ewing's sarcoma: a report of 18 cases and literature review. Chin J Cancer 2010; 29: 420-4.

3. Applebaum MA, Worch J, Matthay KK, Goldsby R, Neuhaus J, West DC, Dubois SG. Clinical Features and Outcomes in Patients with Extraskeletal Ewing Sarcoma. Cancer 2011; 117: 3027-32.

4. Tural D, Molinas Mandel N, Dervisoglu S, et al. Extraskeletal Ewing s Sarcoma Family of Tumors in Adults: Prognostic Factors and Clinical Outcome. Jpn J Clin Oncol 2012; 42: 420-6.
5. Zhu AX, Hong TS, Hezel AF, et al. Current management of gallbladder carcinoma. Oncologist 2010; 15: 168-81.

6. Agarwal N, Kumar S, Sharma S. Synchronous adenocarcinoma of the gall bladder and pancreas in a young woman. Trop Gastroenterol 2013; 34: 50-2.

7. Appukutty SJ, Worthington T, Bagwan IN. Gall bladder malignancy: an unusual association. J Gastrointestin Liver Dis 2013; 22: 217-9.

8. Kim JW, Han JW, Jung SY, Jung JP, Kim JW. Synchronous double primary malignant tumor of the gallbladder and liver: a case report. World J Surg Oncol 2011; 9: 84.

9. Ozan H, Ozerkan K, Aker S, Bülbül M. A case with three primary tumors of the ovary, endometrium and gallbladder. Eur J Gynaecol Oncol 2008; 29: 551-3.

10. Shibuya R, Matsuyama A, Nakamoto M, Shiba E, Kasai T, Hisaoka M. The combination of CD99 and NKX2.2, a transcriptional target of EWSR1-FLI1, is highly specific for the diagnosis of Ewing sarcoma. Virchows Arch 2014; 465: 599-605.

11. Lewis T, Coffin CM, Bernard P. Differentiating Ewing's sarcoma from other round blue cell tumors using a RT-PCR translocation panel on formalin-fixed paraffin-embedded tissues. Modern Pathol 2007; 20: 397-404.

12. Delattre O, Zucman J, Plougastel B, et al. Gene fusion with an ETS DNA-binding domain caused by chromosome translocation in human tumours. Nature 1992; 359: 162-5.

13. Zitelli A, Manfredelli S, Brunotti G, Marcantonio M, Pontone S, Angelici A. Extraskeletal Ewing's sarcoma: insight into a ten years follow-up. Clin Ter 2013; 164: 373-6.

14. Baldini EH, Demetri GD, Fletcher CD, et al. Adults with Ewing's sarcoma/primitive neuroectodermal tumor: adverse effect of older age and primary extraosseous disease on outcome. Ann Surg 1999; 230: 79-86.

15. Wedde TB, Lobmaier IV, Brennhovd B, et al. Primary Ewing's Sarcoma of the Kidney in a 73-Year-Old Man. Sarcoma 2011; 2011: 978319.

16. Huang M, Lucas K. Current therapeutic approaches in metastatic and recurrent Ewing sarcoma. Sarcoma 2011; 2011: 863210.

17. Eckel F, Brunner T, Jelic S; ESMO Guidelines Working Group. Biliary cancer: ESMO Clinical Practice Guidelines for diagnosis, treatment and follow-up. Ann Oncol 2011; 22: 40-4.

18. Valle J, Wasan H, Palmer DH, et al. ABC-02 Trial Investigators: Cisplatin plus gemcitabine versus gemcitabine for biliary tract cancer. N Engl J Med 2010; 362: 1273-81.

\section{Address for correspondence}

Andrzej Plis

Clinic of Hematology and Oncology

Transplant and Palliative Medicine

University Hospital in Greifswald

Sauerbruch St.

Greifswald, Germany

e-mail: andrzejplis@wp.p

Submitted: 5.06 .2014

Accepted: $\quad 7.04 .2015$ 